\title{
High-Sensitive Numerical Gas Detection Using LSPR Effect and Fano Resonance in a Slotted MDM Structure
}

\author{
Hai LIU, Benlei ZHAO, Xu ZHANG, Hancheng ZHANG, \\ Bo WU, and Shoufeng TANG ${ }^{*}$ \\ School of Information and Control Engineering, China University of Mining and Technology, Xuzhou 221116, China \\ ${ }^{*}$ Corresponding author: Shoufeng TANG \\ E-mail: sieedksft@cumt.edu.cn
}

\begin{abstract}
A high-sensitive numerical measurement of methane based on the combined use of the localized surface plasmon resonance (LSPR) and Fano resonance in a slotted metal-dielectric-metal $(\mathrm{MDM})$ periodic structure is numerically investigated. A groove is etched in an original MDM structure to excite the diploe mode at both sides of the groove, and the coherent coupling of two dipole modes is enhanced to realize a fast response, which is beneficial to gas-sensing. The influence of geometric parameters on the reflection spectra and methane sensitivity are analyzed to obtain optimal geometry. Moreover, an etching ring is introduced on the top metal to further raise the coupling area and coupling strength. The Fano resonance is subtly integrated into the optimized structure with asymmetry to achieve greater gas sensitivity. After the introduction of the Fano resonance, the field enhancement caused by the LSPR effect becomes greater and the methane sensitivity can reach up to $8.421 \mathrm{~nm} / \%$ in numerical calculations, which increases $56.8 \%$ more than that of the original one. The combined use of the LSPR and Fano resonance in an optimized MDM structure provides an effective method for high-sensitive gas detection.
\end{abstract}

Keywords: Localized surface plasmon resonance; Fano resonance; gas sensors; MDM structure

Citation: Hai LIU, Benlei ZHAO, Xu ZHANG, Hancheng ZHANG, Bo WU, and Shoufeng TANG, "High-Sensitive Numerical Gas Detection Using LSPR Effect and Fano Resonance in a Slotted MDM Structure," Photonic Sensors, 2022, 12(2): 164-174.

\section{Introduction}

Surface plasmon polariton (SPP) $[1,2]$ is a type of light wave which propagates along with the separation between a dielectric and a metal. Its unique optical characteristics make it overcome the diffraction limit of conventional optical devices. In particular, local surface plasmon resonance (LSPR) [3-5] generally occurs within metallic nanostructures, and the LSPR can effectively control the propagation of free waves and realize the local field electromagnetic enhancement [6, 7]. Since the LSPR is highly sensitive to the dielectric environment's variation, a growing number of
LSPR-based devices have been investigated for various gas-sensing applications. Yang et al. [8,9] reported a methane sensor based on the surface plasmon resonance (SPR) theory, with a sensitivity of $1.078 \mathrm{~nm} / \%$. In 2018, Liu et al. [10] proposed a PCF (photonic crystal fiber)-SPR structure for methane and hydrogen detection, and gas sensitivities were $1.99 \mathrm{~nm} / \%$ and $0.19 \mathrm{~nm} / \%$, respectively. However, the gas-sensitive film is hardly coated onto the inner surface of the PCF-SPR structure, and the diffusion of gas in cladding air holes is relatively slow. Therefore, it is essential to explore an effective sensing method for gas detection to enhance the sensitivity while the coating

Received: 22 December 2020 / Revised: 18 May 2021

(C) The Author(s) 2021. This article is published with open access at Springerlink.com

DOI: $10.1007 / \mathrm{s} 13320-021-0634-5$

Article type: Regular 
fabrication can be simplified. The present sensing principle is mostly based on detecting the resonant peak of spectra. The gas concentration can be calculated through quantifying the shift of the resonant wavelength. Compared with the SPR sensors, the LSPR sensors have outstanding local field enhancement and higher sensitivity to the dielectric environment, which is beneficial for gas detections. The dependence opens up a gas detection approach based on the relationship between the refractive index of gas-sensitive materials and the resonance peak-shifts [11].

Recently, the nanostructures play an important role in designing sensors and absorbers [12-16], especially for the metal-dielectric-metal (MDM) structure. Since the antiparallel surface currents exist on the top and bottom metals, a strong electromagnetic coupling is appropriate for the absorber or sensing systems. In 2017, Liu et al. [17] designed an ultra-wideband absorber based on MDM nanostructure. In the following year, Lei et al. [18] proposed a near-infrared MDM absorber, and the average absorption could reach $97 \%$. In 2020, Bazgir et al. [19] designed a refractive index sensor based on a metal-insulator-metal (MIM) coupled waveguide with a sensitivity of about $1260 \mathrm{~nm} / \mathrm{RIU}$ and figure of merit (FOM) of $120 \mathrm{RIU}^{-1}$. Chao et al. [20] proposed a high-sensitive refractive-index sensor based on the MIM structure with a sensitivity of $3500 \mathrm{~nm} / \mathrm{RIU}$. In 2021, Mahmud et al. [21] designed a plasmonic sensor based on the metal-insulator-metal structure, which was composed of a straight waveguide and a resonator, with a sensitivity of $2713 \mathrm{~nm} / \mathrm{RIU}$ and FOM of 35.1 [21]. Inspired by the reported methods, we use the gas-sensitive film as the dielectric of the MDM structure. The high concentration of the electric field enables the resonant wavelength to have a large shift even though the refractive-index of the gas-sensitive film changes very little. The fabrication technique is much simpler than those of traditional fiber gas-sensors, whose gas-sensitive film is coated on the cladding air-hole.

Because of the high sensitivity of the Fano resonance to a tiny change of surrounding material, the introduction of the Fano resonance provides a new way to enhance the sensitivity $[22,23]$. As is known, the asymmetry can hybridize different plasmon resonances, which promotes the coupling between the wider and narrower plasmon resonances to produce the Fano resonance. The plasmonic modes with net dipole moment can interact with the external electromagnetic field, which is also known as bright modes. However, dark modes with zero net dipole moment radiate extremely weakly, making them hardly interact with the external electromagnetic filed. Notably, the dark modes cannot be excited by light, but they can be excited by the near-filed coupling with bright modes. The dark modes interact with bright modes through the near-field coupling which produces the Fano resonance. Here, the excitement of dark modes and interaction between bright and dark modes can be realized due to the asymmetry of the nanostructure. Compared with local plasmon resonance, the Fano resonance has a reinforcing effect on electromagnetic field enhancement.

In 2019, we [24] proposed an LSPR methane sensor based on a hexagonal gold nanoring array with a sensitivity of $-1.04 \mathrm{~nm} / \%$. However, the sensitivity may not meet the demand for the detections in some harsh circumstances. Highsensitive methane sensors are still needed. Here, an effective method is proposed to enhance the gas sensitivity through the combined use of the MDM structure and Fano resonance. Firstly, we design a simple MDM structure with a slit in the middle dielectric layer and the top metal layer to excite the dipole mode and generate coherent coupling in the grove, which produces a strong electric field. After introducing an etching ring, the proposed sensor achieves a fast response by expanding the reaction area. An increase in the coupling area and intensity is very beneficial to enhance the sensitivity. The 
reason is that the Fano resonance superimposed original plasmon resonance and the electrical field enhancement is also stronger than the previous one. The gas sensitivity under the combined effect of the LSPR and Fano resonance is higher than that under the LSPR effect only. Numerical simulation results show that the maximum methane sensitivity of improved structure can reach up to $8.4214 \mathrm{~nm} / \%$, which further increases $56.8 \%$ than the original value.

\section{Sensing principle and model optimization}

The electron in the noble metal will collectively oscillate locally when the incident light interacts with the nanoparticle. The plasmon resonance peak is dependent on the geometric morphology, size, metal type, and surrounding medium. The dispersion relationship of the LSPR is demonstrated in (1), where $l$ is the depth of penetration, and $\varepsilon_{1}$ and $\varepsilon_{2}$ are the permittivities of $\mathrm{Au}$ and the surrounding medium, respectively. The resonance frequency $w_{s}$ is derived from (2), in which $\omega_{p}$ represents the angular frequency of surface plasmons. Equation(3) implies that the frequency is greatly dependent on the permittivity of the medium illustrated by the Drude-model, in which $\gamma$ corresponds to the damping coefficient of the material. Equation (4) is a result of $\gamma \ll \omega_{p}$ in the region of low frequency. At $\varepsilon_{1}=2 \varepsilon_{m}$, the LSPR frequency $\omega_{\max }$ and LSPR wavelength $\lambda_{\max }$ can be described in (5). Based on the formula $\varepsilon_{m}=n_{m}^{2}$, (6) indicates an equivalent relationship when the frequency is converted to the wavelength. Above all, $\varepsilon_{m}$ and $n_{m}$ represent the dielectric constant and refractive index of the surrounding medium, respectively. $\lambda_{p}$ can be seen as the isochronous elementary frequency of the corresponding bulk metal. As a result, there exists a linear relationship between the plasmon resonance wavelength and the refractive index.

$$
\frac{\varepsilon_{1}}{\varepsilon_{2}}+\frac{l+1}{l}=0
$$

$$
\begin{gathered}
\omega_{s}=\omega_{p} \sqrt{\frac{1}{\varepsilon_{s}(l+1)+1}} \\
\varepsilon_{1}=1-\frac{\omega_{p}^{2}}{\omega^{2}+\gamma^{2}} \\
\varepsilon_{1}=1-\frac{\omega_{p}^{2}}{\omega} \\
\omega_{\max }=\frac{\omega_{p}}{\sqrt{2 \varepsilon_{m}+1}} \\
\lambda_{\max }=\lambda_{p} \sqrt{2 n_{m}^{2}+1} .
\end{gathered}
$$

Figure 1 demonstrates the proposed LSPR sensor structure (Structure A) which is composed of a periodic triple-layer array. Such an MDM array can be fabricated on a clean quartz substrate by the laser holographic lithography technology [25]. Especially, we make the upper metal layer smaller than the middle gas-sensitive layer to increase the reaction area and achieve a faster response. The groove is etched in the top and middle layers to enhance the response efficiency and electric field. Since the two dipole modes on both sides of the groove are coherent, a strong electrical field enhancement occurs in the groove and the middle gas-sensitive film, which provides a potential application in gas sensing. The geometry parameters are defined in Figs. 1(b) and 1(c), where $P$ is the period of the array, $h_{1}$ is the thickness of the top gold film, $h_{2}$ is the thickness of the dielectric spacer, $h_{3}$ is the thickness of the bottom fold film, $d$ is the length of the top square gold, and $w$ is the width of the grove. The dielectric constant of $\mathrm{Au}$ is obtained by the Drude model $\varepsilon=1-\omega_{p}^{2} / \omega\left(\omega+\mathrm{i} \gamma_{0}\right)$, in which the plasmon frequency is $\omega_{p}=1.37 \times 10^{16} \mathrm{~s}^{-1}$ and the collision frequency is $\gamma_{0}=4.08 \times 10^{13} \mathrm{~s}^{-1}$ [26]. The source shape is selected as a periodic plane wave with a polarization vector parallel to the $x$-direction. A series of simulations for the proposed nanostructure is carried out through the finite-difference time domain (FDTD) solution packages. As the proposed nanostructure is periodic, the periodic boundary condition is used in the $x$-direction and $y$-direction while the boundary 
condition in the $z$-direction is the perfect match layer (PML). The sensing characteristics will be acquired by analyzing the plasmon resonance peak-shifts under various geometric parameters.

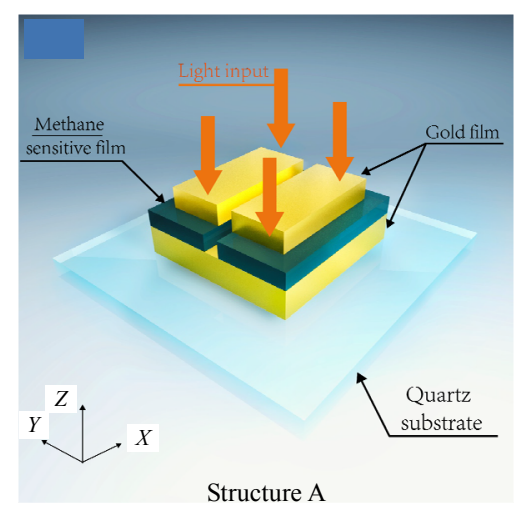

(a)

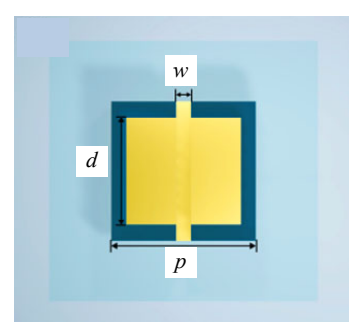

(b)

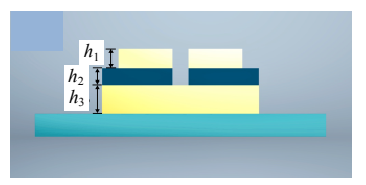

(c)

Fig. 1 Schematic structure of the MDM unit cell: (a) 3-dimemsional (3D) view, (b) top view, and (c) side view of the structure.

The influence of the proposed nanostructure's geometric parameters on sensitivity is analyzed by calculating the reflection peak shifts. In analogous calculations, the thickness of the sensitive film $\left(h_{2}\right)$ always keeps at $10 \mathrm{~nm}$. We change the top metal length $(d)$ from $700 \mathrm{~nm}$ to $780 \mathrm{~nm}$ and vary the thickness of the top metal $\left(h_{1}\right)$ from $10 \mathrm{~nm}$ to $40 \mathrm{~nm}$ with an increment of $10 \mathrm{~nm}$, and an increase in the width of the grove $(w)$ from $10 \mathrm{~nm}$ to $40 \mathrm{~nm}$ to optimize the structure. As the bottom metal can be seen as a mirror, it prohibits the transmission of the electromagnetic, so it is unnecessary to take the thickness of the bottom metal layer into account. The methane-sensitive material can be prepared by using cryptophane A with ultraviolet curable fluoro-siloxane (UVCFS). A dilute solution is applied to the surface of the metal layer, and then the surface of the film is polished with a nitrogen stream of $0.4 \mathrm{MPa}$. It is relatively feasible to coat the gas-sensitive composite film on the underlying metal by the capillary dip layer technique. Different from conventional gas sensors, the proposed sensor structure has higher sensitivity without interference of other gases. The spectral response to the target gas can be enhanced by optimizing the structural parameters. Such gas-sensitive film has a good repeatability and linear relationship between the refractive index $n_{\text {eff }}$ and methane concentration $C_{\mathrm{CH}_{4}}$ within a range from $0 \%$ to $3.5 \%$ [9], as is expressed in (7):

$$
n_{\mathrm{eff}}=1.4478-0.0038 C_{\mathrm{CH}_{4}} \text {. }
$$

Figure 2(a) demonstrates the sensitivity of the individual nanostructure with various values of groove width $(w)$. The influence of the groove width is very little and the sensitivity can reach the maximum when $w=20 \mathrm{~nm}$. Therefore, we single out the groove with a width of $20 \mathrm{~nm}$ for further research at first. Figures 2(b) and 2(c) demonstrate the sensitivity under different values of $d$ and $h_{1}$. Obviously, the gas sensitivity is greatly affected by the metallic square's thickness rather than the length. With an increase in $d$, the local electric field gets enhanced, and the methane sensitivity increases. However, the sensitivity decreases as $h_{1}$ increases. The methane sensitivities under different values $d$ and $h_{1}$ when $w=20 \mathrm{~nm}, h_{2}=10 \mathrm{~nm}$, and $h_{3}=25 \mathrm{~nm}$ are obtained in Table 1 . The methane sensitivity can reach up to $7.30 \mathrm{~nm} / \%$ when we select $d=780 \mathrm{~nm}$ and $h_{1}=30 \mathrm{~nm}$. 


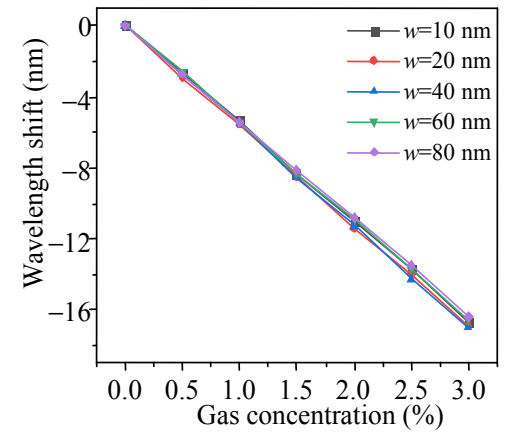

(a)

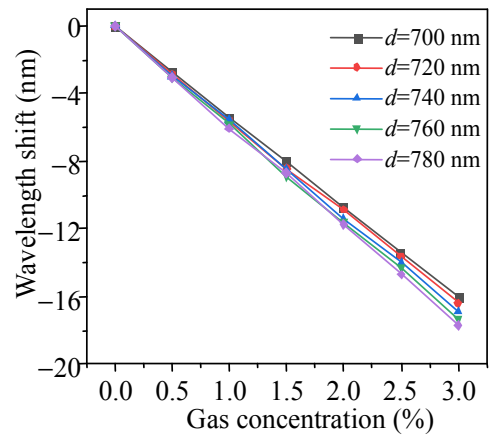

(b)

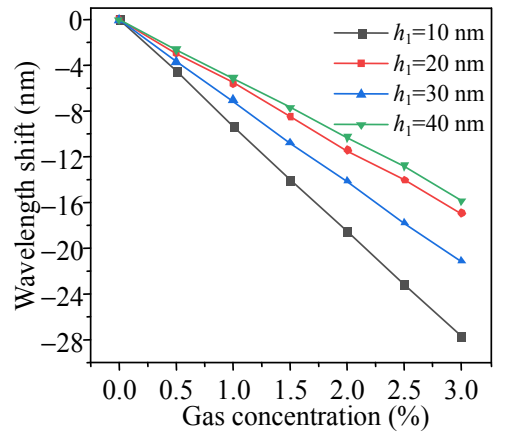

(c)

Fig. 2 Methane sensitivities under different values of (a) slit width, (b) length of top metal, and (c) thickness of top metal.

Table 1 Methane sensitivities under different values of $d$ and $h_{1}$ at $w=20 \mathrm{~nm}$.

\begin{tabular}{cccc}
\hline Group number & $\begin{array}{c}\text { Top length } \\
d(\mathrm{~nm})\end{array}$ & $\begin{array}{c}\text { Top thickness } \\
h_{1}(\mathrm{~nm})\end{array}$ & $\begin{array}{c}\text { Sensitivity } \\
(\mathrm{nm} / \%)\end{array}$ \\
\hline$A_{1}$ & 700 & 20 & 5.35 \\
$A_{2}$ & 740 & 20 & 5.62 \\
$A_{3}$ & 780 & 20 & 5.87 \\
$A_{4}$ & 700 & 30 & 6.64 \\
$A_{5}$ & 740 & 30 & 7.03 \\
$A_{6}$ & $\mathbf{7 8 0}$ & $\mathbf{3 0}$ & 7.30 \\
$A_{7}$ & 700 & 40 & 4.87 \\
$A_{8}$ & 740 & 40 & 5.21 \\
$A_{9}$ & 780 & 40 & 5.24 \\
\hline
\end{tabular}

However, we still need a further optimization for the investigation. We introduce an etching ring with an outer radius $R_{2}=240 \mathrm{~nm}$ and an inner radius $R_{1}=100 \mathrm{~nm}$ into the top metal layer. After introducing an etching ring, the proposed structure achieves a fast response through increasing the reaction area. Since the LSPR generated by the ring resonance cavity enhances the coupling area and intensity, it is very beneficial to improve the sensitivity. The improved structure, schematically demonstrated in Fig. 3, can be formed through nanoring template lithography. Table 2 records the whole calculated results. The results show there is a significant increase in sensitivity compared with the last groups. Accordingly, the improved nanostructure under $d=780 \mathrm{~nm}$ and $h_{1}=30 \mathrm{~nm}$ can achieve the maximum sensitivity of $7.81 \mathrm{~nm} / \%$.

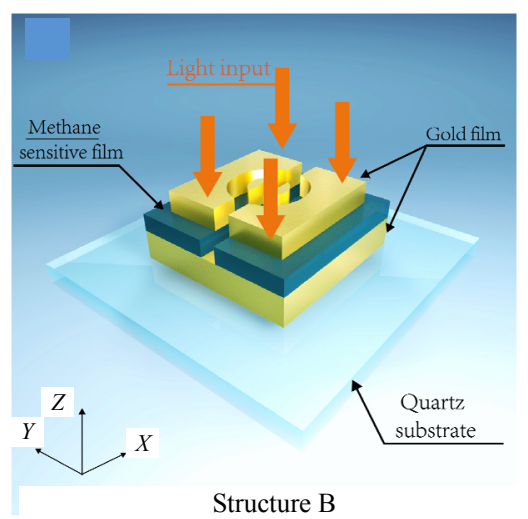

(a)

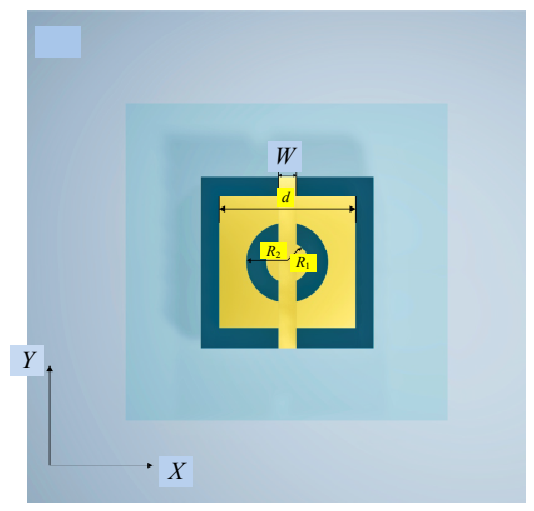

(b)

Fig. 3 Schematic structure of improved structure (Structure B): (a) 3D view and (b) top view. 
Table 2 Methane sensitivities for Structure B and the comparison with Structure A.

\begin{tabular}{ccccc}
\hline $\begin{array}{c}\text { Group } \\
\text { number }\end{array}$ & $\begin{array}{c}\text { Top length } \\
d(\mathrm{~nm})\end{array}$ & $\begin{array}{c}\text { Top thickness } \\
h_{1}(\mathrm{~nm})\end{array}$ & $\begin{array}{c}\text { Sensitivity } \\
(\mathrm{nm} / \%)\end{array}$ & $\begin{array}{c}\text { Increased } \\
\text { rate } \\
(\%)\end{array}$ \\
\hline$B_{1}$ & 700 & 20 & 5.99 & 12.0 \\
$B_{2}$ & 740 & 20 & 6.30 & 11.9 \\
$B_{3}$ & 780 & 20 & 6.43 & 9.5 \\
$B_{4}$ & 700 & 30 & 7.25 & 9.2 \\
$B_{5}$ & 740 & 30 & 7.59 & 7.97 \\
$B_{6}$ & $\mathbf{7 8 0}$ & $\mathbf{3 0}$ & $\mathbf{7 . 8 1}$ & $\mathbf{7 . 0}$ \\
$B_{7}$ & 700 & 40 & 5.23 & 7.4 \\
$B_{8}$ & 740 & 40 & 5.37 & 3.1 \\
$B_{9}$ & 780 & 40 & 5.62 & 7.9 \\
\hline
\end{tabular}

For example, Fig. 4 presents the improved structure's reflection spectra at $d=780 \mathrm{~nm}$, $h_{1}=30 \mathrm{~nm}$, and $w=20 \mathrm{~nm}$, which are corresponding to the mentioned groups $A_{6}$ and

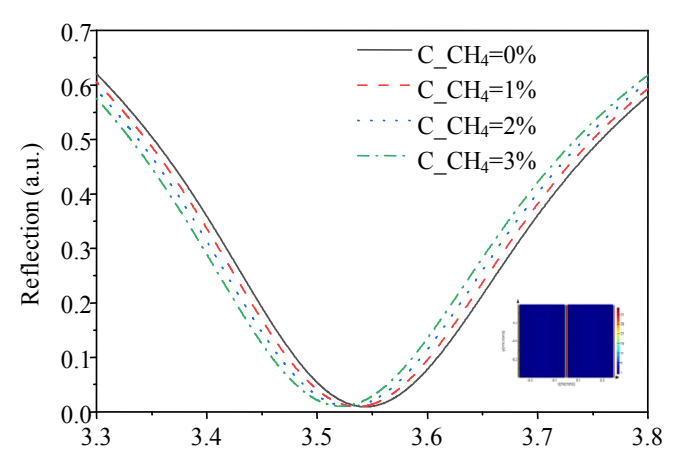

(a)

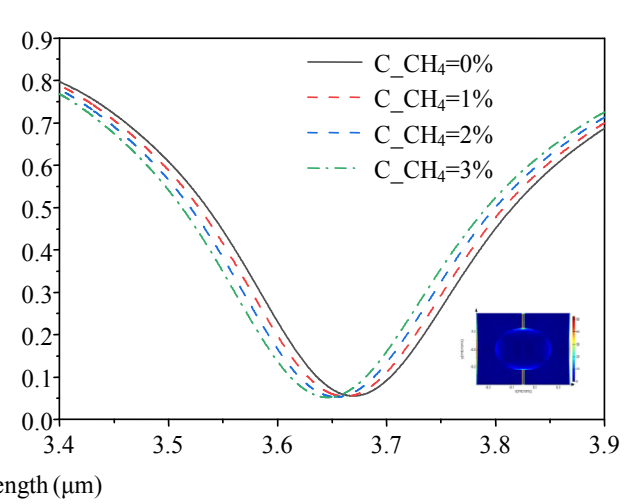

(b)

$B_{6}$. It is clear that the refection intensity of the improved structure is greater than those of Structure A for the majority of plasmon excitation wavelengths, particularly at the plasmon resonance wavelength in Fig. 4. It can be explained that the etching part enables the bottom metal to reflect more light. As is shown in the inserted electric-field distribution in Fig. 4(b), only one resonant peak exists when the electric field distribution profile of the etching ring resonance achieves a dominant point. The electric-field enhancement may arise from the plasmon coherent coupling between the etching ring's inner and outer surfaces [27]. The methane sensitivity increases from $7.30 \mathrm{~nm} / \%$ to $7.81 \mathrm{~nm} / \%$, and the improved structure is beneficial to fast response.

Fig. 4 Optical reflection spectrum of: (a) primary structure $A_{6}$ and (b) improved structure $B_{6}$.

In order to better evaluate the influence of geometric parameters on the sensing performance, we perform additional FDTD simulations for etching rings with different radius. After complete calculations, Table 3 exhibits the sensitivities under different radii and comparison with the structure $B_{6}$. With an increase in $R_{2}$, the methane sensitivity first increases and then gradually decreases. The coupling area becomes larger as the outer radius gets larger, but as the distance increases to a certain value, the coherent coupling between the inner and outer sides becomes attenuated. When $R_{2}=300 \mathrm{~nm}$ and $R_{1}=100 \mathrm{~nm}$, the sensitivity can reach to the maximum of $7.98 \mathrm{~nm} / \%$. In particular, if we choose $R_{2}=300 \mathrm{~nm}$ and $R_{1}=0 \mathrm{~nm}$, the nanoring becomes a nanodisk, and a sensitivity of $7.89 \mathrm{~nm} / \%$ is obtained, which is almost the same as the values above. From the viewpoint of the fabrication technique, the fabrication of the improved structure with a nanodisk is much easier than that case of $R_{2}=300 \mathrm{~nm}$ and $R_{1}=100 \mathrm{~nm}$. Comprehensively considering response speed, electric field strength, and fabrication technique, the structure with a nanodisk of $300 \mathrm{~nm}$ radius is singled out for further investigation. 
Table 3 Methane sensitivity under the different radius of etching ring and the comparison with structure $B_{6}$.

\begin{tabular}{ccccc}
\hline $\begin{array}{c}\text { Group } \\
\text { number }\end{array}$ & $\begin{array}{c}\text { Outer radius } \\
R_{2}(\mathrm{~nm})\end{array}$ & $\begin{array}{c}\text { Inner radius } \\
R_{1}(\mathrm{~nm})\end{array}$ & $\begin{array}{c}\text { Sensitivity } \\
(\mathrm{nm} / \%)\end{array}$ & $\begin{array}{c}\text { Increased rate } \\
(\%)\end{array}$ \\
\hline$C_{1}$ & 280 & 100 & 7.92 & 1.4 \\
$C_{2}$ & 300 & 100 & 7.98 & 2.2 \\
$C_{3}$ & 320 & 100 & 7.71 & -1.3 \\
$C_{4}$ & 340 & 100 & 7.52 & -3.7 \\
$C_{5}$ & $\mathbf{3 0 0}$ & $\mathbf{0}$ & $\mathbf{7 . 8 9}$ & $\mathbf{1 . 0}$ \\
\hline
\end{tabular}

Considering the excellent sensing characteristics of the Fano resonance [28], we intend to introduce the Fano resonance through breaking the nanostructure's symmetry. We add some etching holes on the corner of the top metal of the previous Structure B as shown in Fig. 5 with a fixed radius $r=100 \mathrm{~nm}$. We find that the case of one-hole has a higher sensitivity, which may originate from the highest asymmetry parameter and relative Fano resonance. In asymmetric structures, the line-shape and resonant position of the Fano resonance can be adjusted by the geometry of the structure. This tunability makes the Fano resonance particularly interesting for different sensing applications. As the Fano resonance superimposes the original plasmon resonance, the filed intensity excited by the LSPR increases. The resonant peak will have an obvious shift as gas concentration varies. The sensing performance can be significantly improved. It can be seen from Fig. 6(a) that there exist two modes, which are labeled as Mode 1 and Mode 2. The reflection at the resonant wavelength of Mode 2 is rather small which is more beneficial for the detection. Figure $6(\mathrm{~b})$ proves that a higher gas sensitivity can be achieved. The comparison of gas sensitivity with the symmetric structure $C_{5}$ is listed in Table 4.

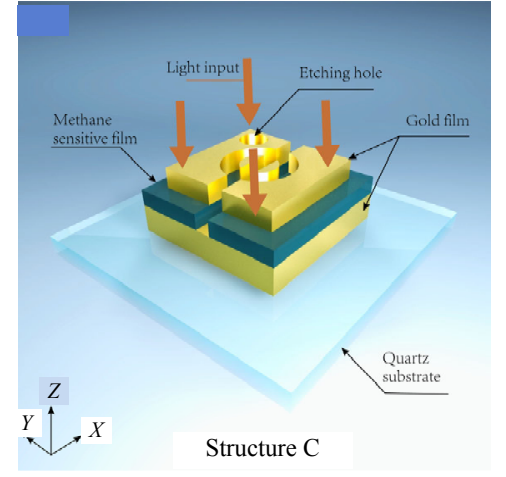

(a)

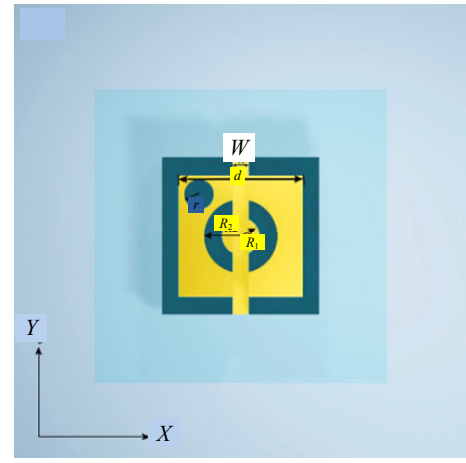

(b)

Fig. 5 Schematic diagram of the asymmetric structure: (a) 3D view and (b) top view.

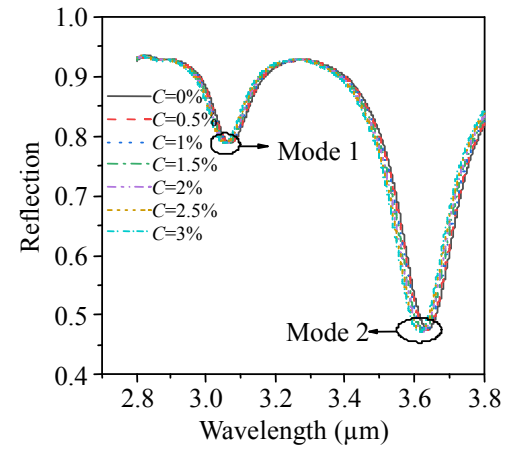

(a)

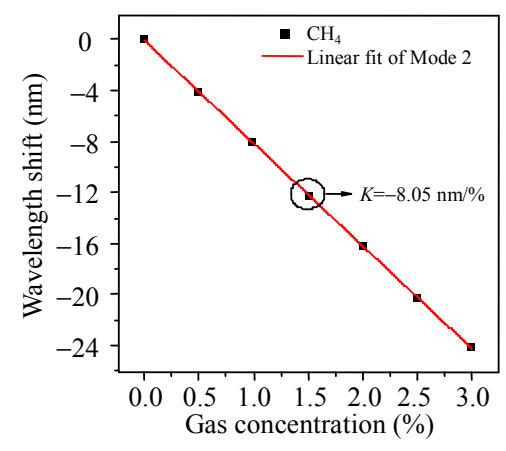

(b)

Fig. 6 Reflection spectra of (a) the asymmetric structure and the wavelength-shifts of (b) methane concentrations. 
Table 4 Comparison between the asymmetric structure and the symmetric structure $\mathrm{C}_{4}$.

\begin{tabular}{cccc}
\hline $\begin{array}{c}\text { Structure } \\
\text { type }\end{array}$ & $\begin{array}{c}\text { Hole radius } \\
r(\mathrm{~nm})\end{array}$ & $\begin{array}{c}\text { Sensitivity } \\
(\mathrm{nm} / \%)\end{array}$ & $\begin{array}{c}\text { Increased rate } \\
(\%)\end{array}$ \\
\hline Symmetric & 0 & 7.52 & 7.0 \\
Asymmetrical & 100 & 8.05 & \\
\hline
\end{tabular}

Obviously, a higher sensitivity of $8.05 \mathrm{~nm} / \%$ can be obtained from this asymmetric structure with only one hole on the left corner. The methane sensitivity increases by $7.0 \%$ compared with the previous one, as is shown in Table 4. It is worth pointing out that the above conclusions are based on the structure $C_{4}$, which corresponds to the minimum value in Table 3. After the introduction of the Fano resonance, the asymmetric structure with the minimum sensitivity can generate a higher sensitivity than the maximum sensitivity in the previous structure. Consequently, for the optimized structure $C_{5}$ that has acquired the maximum sensitivity of $7.89 \mathrm{~nm} / \%$, a final sensitivity of $8.421 \mathrm{~nm} \%$ can be achieved similarly.

\section{Results and expansion}

\subsection{Sensing results}

After comprehensive optimization and the introduction of the Fano resonance for the proposed nanostructure, the methane sensitivity can reach up to $8.4214 \mathrm{~nm} / \%$. Compared with the original structure, the improved structure gains a much better enhancement in sensing performance. According to (7), the refractive index of the sensitive-film has a linear relationship with the target gas within a concentration range of $0 \%-3.5 \%$. In this case, the demodulation formula for single-gas measurement is shown in(8), where $b$ is the intercept, and the gas sensitivity is $k=8.4214 \mathrm{~nm} / \%$.

$$
\Delta \lambda=k \cdot C_{\mathrm{CH}_{4}}+b \text {. }
$$

To assess the accuracy of our demodulation method for different gas concentrations, we select four random testing points [a $(0.3 \%), \mathrm{b}(0.7 \%)$, c (1.8\%), and $\mathrm{d}(2.4 \%)]$ and make comparison with theoretical values obtained by (8). The methane concentration is calculated by analyzing the reflection wavelength shift, as shown in Table5. It presents that the difference between the values of principal values and calculated concentrations is quite small. Therefore, the demodulation method can meet the demand for higher sensitivity and resolution. Table 6 shows the sensitivity comparison between the proposed structure and other reported sensors. Obviously, the sensitivity has a significant improvement. Different from the gas sensors based on the SPR, we adopt an MDM structure to generate the LSPR effect. A great electromagnetic field occurs at the gas-sensitive film due to the antiparallel surface currents between the top and bottom metals, and the electric-field enhancement also occurs in the groove and etching ring. Then, the sensitivity can be improved through such electric-field enhancements. In the proposed MDM structure, the middle dielectric is a methane-sensitive film, which only reacts to methane but not to other gases. The proposed structure has wide applications range and only needs replacing the dielectric with other gas-sensitive films. In fact, such a structure can be extended to an array to measure multiple gases. The selective detection of gas mixtures can be achieved by applying corresponding gas-sensitive films to the array.

Table 5 Values of the calculated results.

\begin{tabular}{ccccc}
\hline $\begin{array}{c}\text { Testing } \\
\text { points }\end{array}$ & $\begin{array}{c}\text { Theoretical } \\
\text { values }\end{array}$ & $\begin{array}{c}\text { Wavelength } \\
\text { shifts }\end{array}$ & $\begin{array}{c}\text { Calculated } \\
\text { value }\end{array}$ & $\begin{array}{c}\text { Errors } \\
\text { rate }\end{array}$ \\
\hline a & 0.7 & -5.73 & 0.6995 & $0.07 \%$ \\
b & 1.4 & -11.43 & 1.3763 & $1.69 \%$ \\
c & 2.2 & -18.26 & 2.1874 & $0.57 \%$ \\
d & 3.1 & -26.19 & 3.1290 & $0.93 \%$ \\
\hline
\end{tabular}

Table 6 Comparison of methane sensitivity to reported methane sensors.

\begin{tabular}{cccc}
\hline No. & Type & Sensitivity $(\mathrm{nm} / \%)$ & Reference \\
\hline 1 & PCF-SPR & 0.540 & Yang et al. $[8]$ \\
2 & PCF-SPR & 1.078 & Yang et al. $[9]$ \\
3 & PCF-SPR & 1.990 & Liu et al. $[10]$ \\
4 & LSPR & 1.040 & Liu et al. [21] \\
5 & PCF-LPG & 2.500 & Yang et al. $[29]$ \\
6 & Proposed sensor & 8.421 & \\
\hline
\end{tabular}




\subsection{Analysis and exploration}

The proposed nanostructure not only can serve as a gas sensor, but also has significant applications in background refractive index measurement. Based on the asymmetric structure, the influence of various dielectric spacers on the background refractive index (RI) sensitivity are investigated, such as $\mathrm{MgF}_{2}$, $\mathrm{Al}_{2} \mathrm{O}_{3}, \mathrm{SiO}_{2}$, and $\mathrm{TiO}_{2}$ [30]. The refractive indices of $\mathrm{MgF}_{2}, \mathrm{Al}_{2} \mathrm{O}_{3}, \mathrm{SiO}_{2}$, and $\mathrm{TiO}_{2}$ are $1.37,1.75,1.4$, and 2.407, respectively. Figure 7 shows the reflection spectra of the MDM structure with $\mathrm{MgF}_{2}, \mathrm{Al}_{2} \mathrm{O}_{3}$, $\mathrm{SiO}_{2}$, and $\mathrm{TiO}_{2}$, respectively. The inserted illustrations are the electric-field distributions of the MDM structure with different dielectrics at the resonant wavelength. The reflection spectra and electric enhanced distribution of the structure with $\mathrm{MgF}_{2}$ are approximatively the same as that case of $\mathrm{SiO}_{2}$. Therefore, the RI sensitivities of the two RI sensors are $554.0 \mathrm{~nm} / \mathrm{RIU}$ and $548.9 \mathrm{~nm} / \mathrm{RIU}$, respectively. Figure 7 also shows that the reflection spectra of the nanostructure with $\mathrm{TiO}_{2}$ have increased to some extent. The intensity of the electric field is weaker than those of $\mathrm{MgF}_{2}$ and $\mathrm{SiO}_{2}$, which is a reason for the lower RI sensitivity. Similarly, the electric field strength of nanostructure with $\mathrm{Al}_{2} \mathrm{O}_{3}$ is also weaker than those of other cases. Figure 8(a) demonstrates that the coupling peak has a red-shift with an increase in the surrounding refractive index. The phenomenon could be effectively explained by the following reasons. The RI increases from 1.34 to 1.37 , a decrease in total dipole component and radiant scattering appears in nanostructure [31, 32].

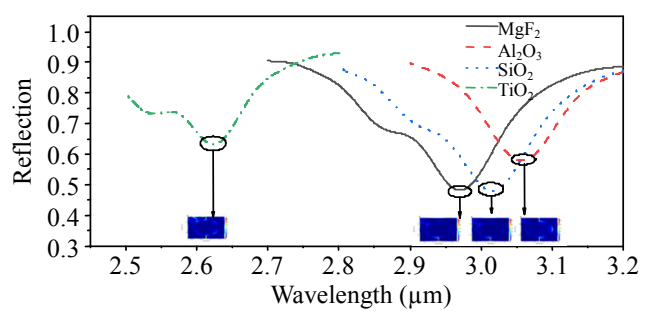

Fig. 7 Reflection spectra of the MDM structure with a dielectric of $\mathrm{MgF}_{2}$ (black line), $\mathrm{SiO}_{2}$ (blue line), $\mathrm{Al}_{2} \mathrm{O}_{3}$ (red line), and $\mathrm{TiO}_{2}$ (green line), respectively. Insert illustration: electric field distribution of MDM structure with four dielectrics at their individual resonant wavelength.

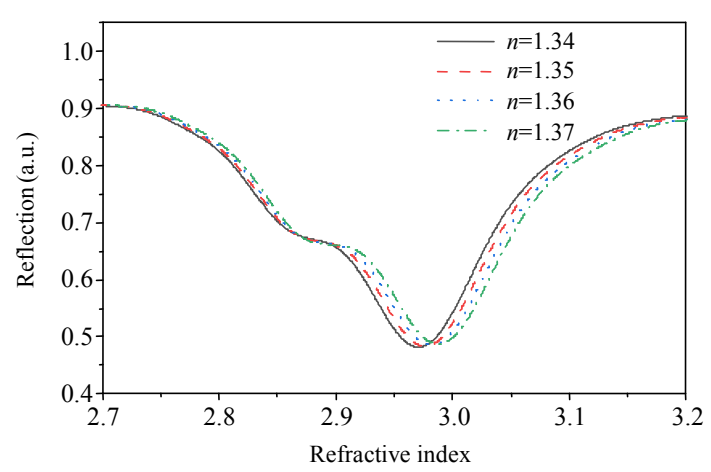

(a)

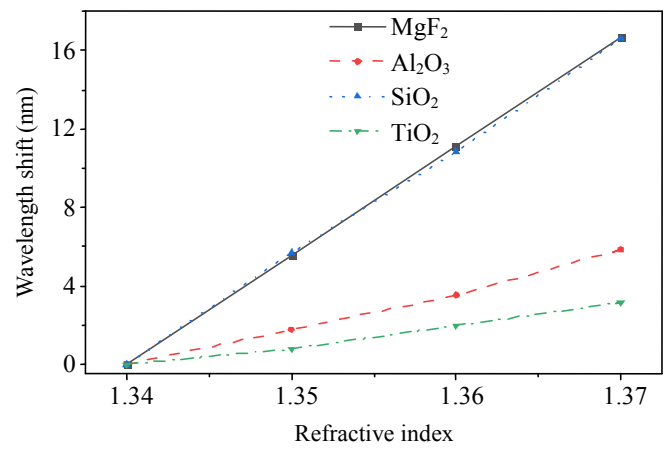

(b)

Fig. 8 Reflection spectra of the asymmetric structure with $\mathrm{MgF}_{2}$ under different background refractive indices (a) and wavelengthshifts of the structure with four different dielectrics (b).

The demodulation formula for the individual sensor with a different spacer is similar to the above methane sensor. Based on the results from Fig. 8(b), the corresponding components of the matrix are demonstrated in Table 7. The RI sensitivity and correlation coefficient of the identical structure with $\mathrm{MgF}_{2}$ are the highest. We also investigate the RI sensitivity of the proposed several structures with $\mathrm{MgF}_{2}$, and the comparison are demonstrated in Table 8. It is discovered that Structure $\mathrm{A}$ at $w=20 \mathrm{~nm}$ obtains a better RI sensing performance $(648 \mathrm{~nm} / \mathrm{RIU})$. This sensing method enables us to have more choices in biosensor and chemical applications.

Table 7 Corresponding elements of the demodulation matrix for four refractive index sensors with different dielectrics.

\begin{tabular}{ccc}
\hline Dielectric type & $\begin{array}{r}\text { RI sensitivity } \\
(\mathrm{nm} / \mathrm{RIU})\end{array}$ & $\begin{array}{c}\text { Correlation coefficient } \\
(\%)\end{array}$ \\
\hline $\mathrm{MgF}_{2}$ & 554.0 & 99.99 \\
$\mathrm{Al}_{2} \mathrm{O}_{3}$ & 193.4 & 99.16 \\
$\mathrm{SiO}_{2}$ & 548.9 & 99.93 \\
$\mathrm{TiO}_{2}$ & 106.3 & 98.80 \\
\hline
\end{tabular}


Table 8 RI sensitivity of the proposed different structures with $\mathrm{MgF}_{2}$.

\begin{tabular}{|c|c|c|c|c|c|}
\hline $\begin{array}{l}\text { Structure } \\
\text { type }\end{array}$ & $\begin{array}{l}\text { Slit width } \\
w(\mathrm{~nm})\end{array}$ & $\begin{array}{c}\text { Outer radius } \\
R_{2}(\mathrm{~nm})\end{array}$ & $\begin{array}{c}\text { Inner radius } \\
R_{1}(\mathrm{~nm})\end{array}$ & $\begin{array}{c}\text { RI } \\
\text { sensitivity } \\
k(\mathrm{~nm} / \mathrm{RI})\end{array}$ & $\begin{array}{c}\text { Correlation } \\
\text { coefficient } \\
R^{2}(\%)\end{array}$ \\
\hline \multirow{2}{*}{ Structure A- } & 0 & 0 & 0 & 308 & 100.00 \\
\hline & 20 & 0 & 0 & 648 & 99.99 \\
\hline \multirow{3}{*}{ Structure B } & 0 & 240 & 100 & 357.6 & 100.00 \\
\hline & \multirow{2}{*}{20} & 240 & 100 & 541.5 & 99.93 \\
\hline & & 300 & 0 & 581.4 & 99.91 \\
\hline Structure C & 20 & 300 & 0 & 554.0 & 99.99 \\
\hline
\end{tabular}

\section{Conclusions}

The plasmon resonance characteristics and sensing performance of an asymmetric MDM structure are systematically investigated by the FDTD method. The sensitivity is substantially improved by optimizing our structure, including adding a ring hole in the center and breaking the structure's symmetry. After a series of optimizing processes, the maximum sensitivity of $8.4214 \mathrm{~nm} / \%$ can be achieved. The proposed method provides a new way for gas detection through the combined use of the MDM structure and Fano resonance. The nanostructure can also be applied to biotechnology and chemical detection as long as the appropriate dielectric is selected as the middle spacer.

\section{Acknowledgment}

Thanks to the State-Level Experimental Teaching Demonstration Center for Electrotechnics and Electronics in CUMT for providing the computing servers. This work was supported by the National Natural Science Foundation of China (Grant No. 51874301) and the Primary Research \& Development Plan of Xuzhou City (Grant No. KC20162).

Open Access This article is distributed under the terms of the Creative Commons Attribution 4.0 International License (http://creativecommons.org/licenses/by/4.0/), which permits unrestricted use, distribution, and reproduction in any medium, provided you give appropriate credit to the original author(s) and the source, provide a link to the Creative Commons license, and indicate if changes were made.

\section{References}

[1] C. Liu, L. Yang, W. Su, F. Wang, T. Sun, Q. Liu, et al., "Numerical analysis of a photonic crystal fiber based on a surface plasmon resonance sensor with an annular analyte channel," Optics Communications, 2017, 382: 162-166.

[2] M. Seifouri, M. A. Rouini, and S. Olyaee, "Design of a surface plasmon resonance biosensor based on photonic crystal fiber with elliptical holes," Optical Review, 2018, 25(5): 555-562.

[3] G. An, S. Li, W. Qin, W. Zhang, Z. Fan, and Y. Bao, "High-sensitivity refractive index sensor based on D-shaped photonic crystal fiber with rectangular lattice and nanoscale gold film," Plasmonics, 2014, 9(6): 1355-1360.

[4] M. Chamanzar, Z. Xia, S. Yegnanarayanan, and A. Adibi, "Hybrid integrated plasmonic-photonic waveguides for on-chip localized surface plasmon resonance (LSPR) sensing and spectroscopy," Optics Express, 2013, 21(26): 32086-32098.

[5] J. Chen, Q. Zhang, C. Peng, C. Tang, X. Shen, L. Deng, et al., "Optical cavity-enhanced localized surface plasmon resonance for high-quality sensing," IEEE Photonics Technology Letters, 2018, 30(8): $728-731$.

[6] S. Tajik and Z. Atlasbaf, "Investigating extraordinary optical transmission and sensing performance through periodic bilayer magnetoplasmonic structure," Journal of Applied Physics, 2020, 127(2): 023102.

[7] S. I. Bozhevolnyi, V. S. Volkov, E. Devaux, J. Y. Laluet, and T. W. Ebbesen, "Channel plasmon subwavelength waveguide components including interferometers and ring resonators," Nature, 2006, 440(7083): 508-511.

[8] J. C. Yang, X. Che, R. Shen, C. Wang, X. M. Li, and W. M. Chen, "High-sensitivity photonic crystal fiber long-period grating methane sensor with cryptophane-A-6Me absorbed on a PAA-CNTs/PAH nanofilm," Optics Express, 2017, 25(17): 20258-20267.

[9] J. C. Yang, L. Zhou, X. Che, J. Huang, X. M. Li, and W. M. Chen, "Photonic crystal fiber methane sensor based on modal interference with an ultraviolet curable fluoro-siloxane nano-film incorporating cryptophane A," Sensors And Actuators B: Chemical, 2016, 235: 717-722.

[10] H. Liu, M. Wang, Q. Wang, H. W. Li, Y. Ding, and C. H. Zhu, "Simultaneous measurement of hydrogen and methane based on PCF-SPR structure with compound film-coated side-holes," Optical Fiber Technology, 2018, 45: 1-7.

[11] P. Vasa, W. Wang, R. Pomraenke, M. Lammers, M. Maiuri, C. Manzoni, et al., "Real-time observation of ultrafast Rabi oscillations between excitons and plasmons in metal nanostructures with J-aggregates," Nature Photonics, 2013, 7(2): 128-132.

[12] S. M. Jiang, D. J. Wu, Y. Cheng, and X. J. Liu, 
"Manipulated localized surface plasmon resonances in silver nanoshells coated with a spherical anisotropic layer," Chinese Physics B, 2012, 21(12): 127806

[13] X. Y. Lu, R. G. Wan, F. Liu, and T. Y. Zhang, "High-sensitivity plasmonic sensor based on perfect absorber with metallic nanoring structures," Journal of Modern Optics, 2016, 63(2): 177-183.

[14] X. Q. Xu, X. L. Hu, X. S. Chen, Y. S. Kang, Z. P. Zhang, K. B. Parizi, et al., "Engineering a large scale indium nanodot array for refractive index sensing," ACS Applied Materials \& Interfaces, 2016, 8(46): 31871-31877.

[15] S. A. Wang, X. H. Sun, M. J. Ding, G. D. Peng, Y. L. Qi, Y. L. Wang, et al., "The investigation of an LSPR refractive index sensor based on periodic gold nanorings array," Journal of Physics D: Applied Physics, 2018, 51(4): 045101.

[16] S. B. Malani and P. Viswanath, "Impact of ordering of gold nanohole arrays on refractive index sensing," Journal of the Optical Society of America B-Optical Physics, 2018, 35(10): 2501-2508.

[17] T. J. Liu and J. Takahara, "Ultrabroadband absorber based on single-sized embedded metal-dielectricmetal structures and application of radiative cooling," Optics Express, 2017, 25(12): A612-A627.

[18] L. Lei, S. Li, H. Huang, K. Tao, and P. Xu, "Ultra-broadband absorber from visible to near-infrared using plasmonic metamaterial," Optics Express, 2018, 26(5): 5686-5693.

[19] M. Bazgir, M. Jalalpour, F. B. Zarrabi, and A. S. J. J. O. E. M. Arezoomand, "Design of an optical switch and sensor based on a MIM coupled waveguide using a DNA composite," Journal of Electronic Materials, 2020, 49(3): 2173-2178.

[20] C. T. Chou Chao, Y. F. Chou Chau, and H. P. Chiang, "Highly sensitive metal-insulator-metal plasmonic refractive index sensor with a centrally coupled nanoring containing defects," Journal of Physics D: Applied Physics, 2021, 54(11): 115301.

[21] R. A. Mahmud, M. O. Faruque, and R. H. J. O. C. Sagor, "A highly sensitive plasmonic refractive index sensor based on triangular resonator," Optics Communications, 2021, 483: 126634.

[22] R. Singh, I. Al-Naib, W. Cao, and C. Rockstuhl, "The Fano resonance in symmetry broken terahertz metamaterials," IEEE Transactions on Terahertz Science \& Technology, 2013, 3(6): 820-826.

[23] J. Q. Wang, C. Z. Fan, J. N. He, P. Ding, E. J. Liang, and Q. Z. Xue, "Double Fano resonances due to interplay of electric and magnetic plasmon modes in planar plasmonic structure with high sensing sensitivity," Optics Express, 2013, 21(2): 2236-2244.

[24] H. Liu, C. Chen, Y. Zhang, B. Bai, and S. Tang, "A high-sensitivity methane sensor with localized surface plasmon resonance behavior in an improved hexagonal gold nanoring array," Sensors, 2019, 19(21): 4803.

[25] S. Wang, X. H. Sun, W. Y. Li, W. Liu, L. Jiang, and J. Han, "Fabrication of photonic quasicrystalline structures in the sub-micrometer scale," Superlattices and Microstructures, 2016, 93: 122-127.

[26] B. F. Chen, C. S. Lee, and R. L. Elsberry, "On tropical cyclone size and intensity changes associated with two types of long-lasting rainbands in monsoonal environments," Geophysical Research Letters, 2014, 41(7): 2575-2581.

[27]X. He, N. O'Keefe, Y. J. Liu, D. C. Sun, H. Uddin, A. Nirmalathas, et al., "Transmission enhancement in coaxial hole array based plasmonic color filter for image sensor applications," IEEE Photonics Journal, 2018, 10(4): 1-9.

[28] B. Luk'yanchuk, N. I. Zheludev, S. A. Maier, N. J. Halas, P. Nordlander, H. Giessen, et al., "The Fano resonance in plasmonic nanostructures and metamaterials," Nature Materials, 2010, 9(9): 707-715.

[29] J. Yang, L. Zhou, J. Huang, C. Tao, X. Li, and W. Chen, "Sensitivity enhancing of transition mode long-period fiber grating as methane sensor using high refractive index polycarbonate/cryptophane A overlay deposition," Sensors \& Actuators B: Chemical, 2015, 207: 477-480.

[30] J. Kischkat, S. Peters, B. Gruska, M. Semtsiv, and W. T. Masselink, "Mid-infrared optical properties of thin films of aluminum oxide, titanium dioxide, silicon dioxide, aluminum nitride, and silicon nitride," Applied Optics, 2012, 51(28): 6789 6798.

[31] J. Aizpurua, P. Hanarp, D. S. Sutherland, M. K1l, G. W. Bryant, and G. D. A. Fj, "Optical properties of gold nanorings," Physical Review Letters, 2003, 90(5): 057401.

[32] N. Liu, M. Mesch, T. Weiss, M. Hentschel, and H. Giessen, "Infrared perfect absorber and its application as plasmonic sensor," Nano Letters, 2010, 10(7): 2342-2348. 